\title{
1 Einleitung
}

Das Theater ist eine gesellschaftliche Institution, in der vor einem Publikum erprobt wird, was sozial möglich ist: Es schafft eine soziale Situation, in der es darum geht, die Neugier und die Urteilskraft der Zuschauer zu wecken und sie dazu zu bewegen, sich darauf einzulassen, was auf der Bühne passiert, es sich anzuschauen, anzuhören und es auszuhalten (vgl. Baecker 2005: 10). Das Theater ist der Ort, an dem die Gesellschaft sich im Spiegel ihrer selbst betrachten kann, an dem „die Kommunikation der Gesellschaft sich der Kommunikation der Gesellschaft präsentiert“ (Baecker 2005: 14). Da es zumindest in der westlichen Theaterpraxis nicht üblich und angebracht ist, sich während der Theatervorstellung über das Rezipierte zu verständigen (vgl. Linz 2018: 203-204), kann der Zuschauer die Frage, wie er zu dem steht, was ihm auf der Bühne präsentiert wird, möglicherweise vorerst nur für sich selbst beantworten (vgl. Krotz 1997: 101). Es bleibt aber in der Regel nicht bei einer rein passiven Rezeption durch den individuellen Betrachter, sondern neben der Theateraufführung gehört auch die Theaterpause als fester Bestandteil zur sozialen Veranstaltung des Theaterbesuchs. Mit der Theaterpause bietet das Theater als gesellschaftliche Institution den Besuchern Infrastrukturen zur Erholung und ermöglicht ihnen die Aneignung der im Theaterstück präsentierten Inhalte in der geselligen Interaktion mit anderen im Anschluss an die Rezeption. Erst aus dieser Anschlusskommunikation geht hervor, inwiefern das Theaterstück Neugier und Urteilskraft der Zuschauer weckt, inwieweit sie das, was ihnen auf der Bühne präsentiert wird, aushalten können, und in welchem Maße sie die im Theaterstück präsentierte Welt mit der Welt außerhalb des Theaterstücks, das heißt mit ihrer eigenen Alltagswirklicheit und der gesellschaftlichen Wirklichkeit, in Beziehung setzen.

Wie extensiv die Beteiligten das Pausengespräch als Anschlussmöglichkeit an den Rezeptionsprozess nutzen, um sich über den Abgleich von Wahrnehmungen und Positionen das im Theaterstück Rezipierte anzueignen und so einen Beitrag zur Kunstkommunikation zu leisten, und inwieweit sie damit zur Konstitution des Theaters beitragen, soll im Rahmen der vorliegenden Untersuchung geklärt werden. Damit verbunden ist ebenso die Frage, in welchem Maße die Beteiligten die semi-öffentliche Kommunikationssituation auch als Bühne nutzen, um sich in der geselligen Interaktion mit anderen selbst als Kunstkenner oder -kritiker darzustellen und zu positionieren und sich so möglicherweise mittels spezifischer kommunikativer und (bildungs)sprachlicher Praktiken - durch die Wahl bestimmter „Redeweisen“ (Müller/Kluwe 2012: 3) - sozial von anderen zu unterscheiden. 
Prozesse dieser sozialen Positionierungen lassen sich nach Müller und Kluwe (2012: 5) besonders gut in der Kunstkommunikation nachvollziehen,

weil die Kunst ein Kommunikationsraum mit außerordentlich hohem Prestige ist: Der hohe soziale Distinktionswert der Kunst ermöglicht für sprachlich Handelnde im künstlerischen Kommunikationsbereich per se die Möglichkeit der sozialen Positionierung, da Wissen über Kunst immer ein exklusives Wissen ist.

(Müller/Kluwe 2012: 5, Herv. im Original)

Laut Hausendorf (2012: 95) werden soziale Positionierungen in der Kunstkommunikation von den Beteiligten typischerweise im Zuge des Bewertens vorgenommen, dem insgesamt als eine zentrale Aufgabe (neben dem Bezugnehmen, Erläutern, Beschreiben und Deuten) in diesem Kontext ein hoher Stellenwert zukommt. Linguistische Untersuchungen von Bewertungen in der mündlichen Alltagskommunikation über Kunst (vgl. unter anderem Hausendorf 2005, 2007, 2011, 2012 sowie Hausendorf/Müller 2016) erfolgten bisher vor allem mit Bezug auf bildende Kunst, etwa auf Basis von Gesprächen, die parallel zur Betrachtung eines Kunstwerks bei einem gemeinsamen Museumsbesuch geführt wurden.

Neben dem Bereich der Kunstkommunikation wurden Bewertungen bereits in verschiedenen anderen Kommunikationsbereichen linguistisch untersucht, etwa in der Arzt-Patienten-Kommunikation (vgl. zum Beispiel Fiehler 2005; Heath 1992; Spranz-Fogasy 2014) oder in der politischen Kommunikation (vgl. zum Beispiel Girnth 1993; Reisigl 2008). Auch in der Erforschung sozialer Interaktionssituationen in der Konversationsanalyse spielte und spielt das Bewerten nach wie vor eine wichtige Rolle. Frühe Studien zu Bewertungsinteraktionen in privaten Alltagsgesprächen (vgl. unter anderem Pomerantz 1975, 1984; Auer/Uhmann 1982 sowie Goodwin/Goodwin 1992) stellen allgemeine sequenzielle Strukturen von Bewertungsinteraktionen und die Präferenz für Übereinstimmung als durchgängiges konversationelles Prinzip heraus. Jüngere Studien, die in einem von Lindström und Mondada (2009) herausgegebenen Sonderheft zu Bewertungen in der sozialen Interaktion zusammengetragen worden sind, geben Aufschluss über einige institutionsspezifische Ausprägungen von Bewertungsinteraktionen und relativieren den in manchen konversationsanalytischen Untersuchungen privater Alltagskommunikation konstatierten Allgemeingültigkeitsanspruch konversationeller Prinzipien. So zeigen die im oben genannten Sonderheft versammelten Beiträge auf Basis multimodaler Analysen unter anderem, dass Bewertungen sowohl mit Bezug zum jeweiligen Kontext realisiert werden als auch den Kontext konstituieren und aktualisieren und dass eine Präferenz für Übereinstimmung nicht notwendigerweise gegeben ist. 
Obwohl das Bewerten in linguistischen Untersuchungen verschiedener Kommunikationsbereiche, in der Interaktion in privaten Alltagsgesprächen und in einigen institutionellen Kontexten in der konversationsanalytischen Forschung bereits ausführlich untersucht wurde und seit einigen Jahren auch einen Untersuchungsfokus der linguistischen Analyse von Kunstkommunikation darstellt, wurde bisher noch nicht systematisch erforscht, wie das Bewerten im Rahmen der gemeinsamen Rezeption performativer Kunst im Theater in der nicht-öffentlichen beziehungsweise semi-öffentlichen Alltagskommunikation (vgl. Hausendorf/Müller 2016: 43) in Pausengesprächen von den Beteiligten realisiert wird. ${ }^{1}$ Eine solche Untersuchung von Bewertungsinteraktionen in Pausengesprächen im Theater ist gerade deshalb interessant, weil bei den Pausengesprächen situative Gegebenheiten vorliegen (vgl. auch Habscheid/Linz 2018), die sich grundlegend von der Kommunikation im Museum unterscheiden: Bei einem Theaterbesuch sind Rezeptions- und Kommunikationsprozess entkoppelt. Erst das Pausengespräch als fester Bestandteil der Institution des Theaters schafft Ermöglichungsbedingungen für gesellige Interaktion und den Austausch über durch das Theaterstück angestoßene gesellschaftliche Fragen. Es bietet den Beteiligten Entlastung von einer möglicherweise anstrengenden Rezeption(serfahrung) und von mit der Kommunikation über das Theaterstück verbundenen Zugzwängen, wobei die kommunikative Bearbeitung von im Theaterstück rezipierten Inhalten und die gesellige Interaktion fließend ineinander übergehen (vgl. Habscheid/Linz 2018: 4).

Der Frage, wie das Bewerten im Rahmen der gemeinsamen Rezeption performativer Kunst im Theater in der semi-öffentlichen Alltagskommunikation von den Beteiligten in den Pausengesprächen interaktiv realisiert wird, nimmt sich die vorliegende Arbeit an. Ziel der Arbeit ist es, spezifische Ausprägungen von Bewertungsinteraktionen in an verschiedenen Theatern erhobenen Pausengesprächen herauszustellen, die von Zuschauern im Anschluss an die Rezeption eines Theaterstücks oder in der Pause zwischen den Teilen eines Theaterstücks geführt wurden. Mit dieser Untersuchung leistet die Arbeit einerseits einen Beitrag $\mathrm{zu}$ einem bisher in der Konversationsanalyse noch nicht untersuchten

1 Eine systematische Untersuchung von sprachlich-kommunikativen Alltagspraktiken des Publikums im Foyer- beziehungsweise Pausengespräch wurde im von 2014 bis 2016 von der DFG geförderten Projekt Theater im Gespräch. Sprachliche Kunstaneignungspraktiken in der Theaterpause unter der Leitung von Stephan Habscheid (Universität Siegen) und Erika Linz (Universität Bonn) durchgeführt. Die Ergebnisse wurden in einem Sammelband mit dem Titel Theater im Gespräch. Sprachliche Publikumspraktiken in der Theaterpause (vgl. Gerwinski/Habscheid/ Linz 2018) veröffentlicht. 
Bereich semi-öffentlicher institutioneller Kommunikation, andererseits gibt sie Aufschluss über die Relevanz des Bewertens für die Aneignung der im Theaterstück präsentierten Inhalte und damit auch über die Relevanz und Funktion(en) des Pausengesprächs für das Publikum und die Konstitution des Theaters. Eine gesprächsanalytische Vorgehensweise erweist sich dabei insofern als geeignet, als dass sie ein ideales Instrumentarium für eine differenzierte Untersuchung der Spezifika, Kontingenzen und Dynamiken der interaktiven Realisierung von Bewertungen bereitstellt.

Das theoretische Fundament ist durch den Untersuchungsfokus sowie die Datengrundlage dieser Arbeit bestimmt. Um die Frage nach spezifischen Ausprägungen von Bewertungsinteraktionen in Pausengesprächen im Theater(foyer) beantworten zu können, werden in Kapitel 2 zuerst linguistische Ansätze und Modelle zur Beschreibung von Bewertungen aus handlungstheoretischer und kontrastierend aus praxeologischer Perspektive sowie Bewertungsinteraktionen im Allgemeinen und mit Bezug zu institutionellen Kontexten aus gesprächsanalytischer Perspektive diskutiert und für die spätere Analyse von Bewertungsinteraktionen relevante Begrifflichkeiten vorgestellt. Außerdem werden mit dem Bewerten verbundene Aspekte und Konzepte erläutert. Hierzu gehören das Indizieren und Aushandeln von Wissen, Haltungen (Stancetaking), Selbst- und Fremdpositionierungen sowie von Face.

In Kapitel 3 wird ein Überblick über den Forschungsstand zum Bewerten als Gegenstand in der Forschung zur Aneignungskommunikation und in der Publikumsforschung (audience studies) gegeben. Untersuchungen zur Anschlussbeziehungsweise Zuschauerkommunikation sind insofern für die Untersuchung der Pausengespräche in der vorliegenden Arbeit relevant, als die an den Pausengesprächen Beteiligten als Teil eines räumlich und zeitlich ko-präsenten Publikums nach der Rezeption des Theaterstücks oder nach der Rezeption zumindest eines Teils des Theaterstücks durch einen interaktiven Abgleich des Erlebten Anschlusskommunikation über (performative) Kunst betreiben und sich so die Inhalte des Theaterstücks kommunikativ aneignen können. Vor allem bisherige Forschungsarbeiten zur Zuschauerkommunikation während des gemeinsamen Fernsehens sind als Ausgangspunkt und Vergleichsbasis für die in dieser Arbeit durchgeführte Analyse der Pausengespräche nutzbar. Des Weiteren knüpft die vorliegende Arbeit an bisherige Arbeiten zur Kunstkommunikation an, in denen Gespräche im Rahmen der Betrachtung eines kopräsenten Kunstwerks im Museum untersucht wurden und in denen der besondere Stellenwert von Bewertungen in diesem Kontext herausgestellt wurde. Mit einem abschließenden Blick auf Ergebnisse, die aus Studien zur Untersuchung von Publika und deren Rezeptionserfahrungen, unter anderem mit Bezug auf Bewertungen, aus dem Bereich der Publikumsforschung hervorgegangen sind, wird schließlich 
auch die Perspektive der Soziologie als für die in dieser Arbeit durchgeführte Analyse relevante Vergleichsbasis einbezogen.

Die einen Teil der sozialen Veranstaltung des Theaterbesuchs darstellende Theaterpause zwischen den Teilen eines Theaterstücks oder im Anschluss an das Theaterstück ermöglicht es den Zuschauern, sich das Rezipierte gemeinsam anzueignen und sich gegenüber dem Theaterstück und damit auch gegenüber den anderen Gesprächsteilnehmern zu positionieren sowie über Themen zu sprechen, die keinen unmittelbaren Bezug zum Theaterstück haben, sondern die im Rahmen geselliger Interaktion der Beziehungsgestaltung dienen. Aus diesem Grund werden in Kapitel 4 auf Basis der Forschungsliteratur zu Spezifika geselliger Interaktion die Konzepte ,phatische Kommunion“, ,Small Talk“ und ,homileïscher Diskurs‘ vorgestellt. Da aufgrund des Exklusivitätscharakters der Kunstkommunikation von den Beteiligten durch Bewertungen vorgenommene Positionierungen potenziell heikel sind, kann der Theaterpause insofern eine Entlastungsfunktion zukommen, als dass sie unter anderem Themenmaterial bietet, das von den Beteiligten als Ressource genutzt werden kann, wenn die Kommunikation über den künstlerischen Gegenstand (im weiteren Sinne) zur Belastung wird. Deshalb steht in diesem Kapitel ebenfalls die Frage im Fokus, wie die Beteiligten den Kontext der geselligen Interaktion in ihren Gesprächen relevant setzen und wie die Situation, das heißt die räumlichen und zeitlichen Rahmenbedingungen der Gespräche, von den Beteiligten als kommunikative Ressource genutzt wird, um mit Face-Bedrohungen im Zusammenhang mit dem Bewerten im Kontext der Kunstkommunikation umzugehen.

Die Darlegung der methodischen Basis erfolgt in Kapitel 5. Es werden Grundsätze und Vorgehensweisen der aus der Ethnomethodologie hervorgehenden ethnomethodologischen Konversationsanalyse beziehungsweise Gesprächsanalyse erläutert und für die Nachvollziehbarkeit der in dieser Arbeit präsentierten Transkripte relevante Notationskonventionen vorgestellt.

Die Datengrundlage der vorliegenden Arbeit wird in Kapitel 6 beschrieben. Neben einer knappen Vorstellung des Projekts Theater im Gespräch. Sprachliche Kunstaneignungspraktiken in der Theaterpause, in dessen Rahmen die Gespräche der Theaterbesucher erhoben wurden, werden die Erhebungsorte sowie die Durchführung der Datenerhebung beschrieben. In diesem Zuge wird - im Zusammenhang mit der Frage nach der Authentizität von Daten in gesprächsanalytischen Studien - zudem ausführlicher begründet, warum aufgrund einer möglichst wenig invasiven Gestaltung des Erhebungsdesigns auf Videoaufnahmen verzichtet wurde und sich die Untersuchung stattdessen auf Audioaufnahmen stützt. Das Gesamtdatenkorpus und die für die Untersuchung in dieser Arbeit ausgewählten Gespräche werden im Anschluss vorgestellt, bevor Inhalte der für die vorliegende Arbeit ausgewählten Theaterstücke erläutert 
werden sowie ein Überblick über die Konstellationen der an den ausgewählten Gesprächen beteiligten Zuschauergruppen gegeben wird.

Verbunden mit in der Forschungsliteratur zum Bewerten herausgestellten sprachlichen Mitteln werden in Kapitel 7 auf Basis erster Analysen der dieser Arbeit zugrunde liegenden Gespräche kommunikative Verfahren und sprachliche Mittel präsentiert, die von den an den Bewertungsinteraktionen beteiligten Personen genutzt werden, um Bewertungen $\mathrm{zu}$ realisieren, zu begründen und auszuhandeln. Dieses Kapitel bietet gleichzeitig eine Grundlage für die in einem nächsten Schritt durchgeführte mikroanalytische Untersuchung der sequenziellen Strukturen des interaktiven Vollzugs der Aktivität des Bewertens, in dem die in diesem Kapitel aufgezeigten Verfahren und Mittel zum Tragen kommen.

In Kapitel 8 steht die Rekonstruktion von Charakteristika des interaktiven Vollzugs von Bewertungen im Fokus. Im Rahmen einer kleinschrittigen Analyse der sequenziellen Strukturen von Bewertungsinteraktionen werden zunächst die von den Beteiligten genutzten Möglichkeiten der Eröffnung von Bewertungsinteraktionen in den Blick genommen. An die Betrachtung möglicher Einstiege in die Bewertungsinteraktion schließt sich die Beschreibung sequenzieller und thematischer Verläufe von Bewertungsinteraktionen an. Bei der Beschreibung dieser sequenziellen und thematischen Verläufe von Bewertungsinteraktionen steht die Frage im Fokus, wie die Beteiligten im Zuge des gemeinsamen Hervorbringens und Aushandelns von Bewertungen Bewertungsgegenstände auf der inhaltlichen, der ästhetisch-performativen oder der globalen Ebene des Theaterstücks sowie auf der gesellschaftlichen Ebene außerhalb des Theaterstücks (ko-)konstruieren und sich so das im Theaterstück Präsentierte kommunikativ aneignen. Im Anschluss an die Beschreibung sequenzieller und thematischer Verläufe von Bewertungen werden Formen der Realisierung nicht übereinstimmender Bewertungen und die Aushandlung von Nichtübereinstimmung in Bewertungsinteraktionen in den Blick genommen. In diesem Zusammenhang stellt der in Kapitel 4 dargestellte gesellige Kontext eine wichtige Ressource für das Management divergierender Positionierungen hinsichtlich des Theaterstücks oder einiger Teilaspekte des Theaterstücks dar. In einem letzten Schritt erfolgt unter Berücksichtigung der semi-institutionellen Rahmenbedingungen der Theaterpausengespräche die Darstellung möglicher Formen der Beendigung von Bewertungsinteraktionen.

Die im Rahmen der Analyse herausgestellten Charakteristika von Bewertungsinteraktionen in Pausengesprächen im Theater(foyer) werden in einer Schlussbetrachtung in Kapitel 9 zusammengefasst, auf die im theoretischen Teil der Arbeit dargestellten Ergebnisse rückbezogen und diskutiert. Neben der Zusammenfassung der Ergebnisse werden in diesem Kapitel in einem Ausblick mögliche weitere, an diese Arbeit anknüpfbare Untersuchungen aufgezeigt. 